EPJ Web of Conferences 41, 04001 (2013)

DOI: $10.1051 /$ epjconf/20134104001

C) Owned by the authors, published by EDP Sciences, 2013

\title{
Quantum-Optical Spectroscopy of Semiconductors
}

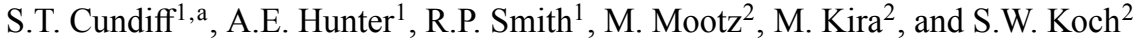 \\ 1 JILA, University of Colorado and National Institute of Standards and Technology, Boulder, Col- \\ orado, 80309-0440 USA \\ 2 Department of Physics, Philipps University Marburg, Renthof 5, D-35032 Marburg, Germany
}

\begin{abstract}
We analyze non-linear absorption spectra of semiconductor quantum wells and use a cluster-expansion transformation to project a large set of quantitative classical measurements onto the true quantum responses. Classical and quantum responses yield significantly different results.
\end{abstract}

Laser spectroscopy has been highly successful at probing the structure and dynamics of matter. The most sensitive spectroscopic methods often rely on differential measurements where a parameter is modulated, for example the laser's intensity or frequency, and a corresponding modulation in the light-matter interaction is observed. A logical, but challenging, extension of this idea is to modulate the quantum optical statistics of the light and study any resulting modulation in the response of the material under study [1]. Currently this cannot be directly implemented in the laboratory because high-intensity sources with arbitrary and controllable quantum statistics are not available.

To overcome this difficulty, we use a hybrid approach combining experiment and theory [2]. We perform careful, quantitative measurements of the response of a system to a classical light field, namely a coherent state $|\beta\rangle$, as the strength of the coherent state, $\beta$, is scanned. Using this data, we then perform a cluster expansion transformation (CET) [3] to project how the system would respond to light with non-classical quantum statistics.

To apply our approach to semiconductors, we experimentally measure the absorption at a fixed delay of 13 ps from ten GaAs quantum wells (QWs) in a cross-circular pump-probe configuration. Performing careful calibration, we record the absorption quantitatively as a function of probe-photon energy, $E$, and pump $|\beta\rangle$. As the pump and probe have orthogonal polarization directions, pumpgenerated coherences cannot produce phase-sensitive contributions to the probe response. As a result, the measured absorption depends only on the intensity of the pump, such that one needs to perform only a one-dimensional phase-space sweep in terms of $|\beta\rangle$. Here, the largest $\beta$ used creates more than one million interacting electrons and holes, such that the absorption spectrum characterizes the optical response of a highly nontrivial many-body system.

The recorded classical absorption spectra are shown in Fig. 1. For weak $|\beta\rangle$, the spectra exhibit the lowest resonance at the excitonic $1 \mathrm{~s}$ heavy-hole energy, $E_{1 S}=1.547 \mathrm{eV}$, and the second resonance appears at the $1 \mathrm{~S}$ light-hole position, $6.90 \mathrm{meV}$ above $E_{1 S}$. As the pump and probe have opposite circular polarizations, a further resonance develops at $2.25 \mathrm{meV}$ below $E_{1 S}$ for $\beta$ above 1,000 . The emergence of this spectral feature is related to the formation of a biexciton. For large $\beta$, we clearly see that the absorption spectrum exhibits strong many-body nonlinearities due to excitation-induced dephasing effects, the subtle features of which can even be used to identify the electron-hole and exciton distributions of the excited QWs [4].

The constructed CET distributions follow the measured dependence of the absorption coefficient on the pump intensity extremely accurately. Therefore, we can reliably apply CET projection to produce the differential absorption for any single-photon perturbations. More specifically, we construct

\footnotetext{
a e-mail: cundiff@jila.colorado.edu
}

This is an Open Access article distributed under the terms of the Creative Commons Attribution License 2.0, which permits unrestricted use, distribution, and reproduction in any medium, provided the original work is properly cited. 


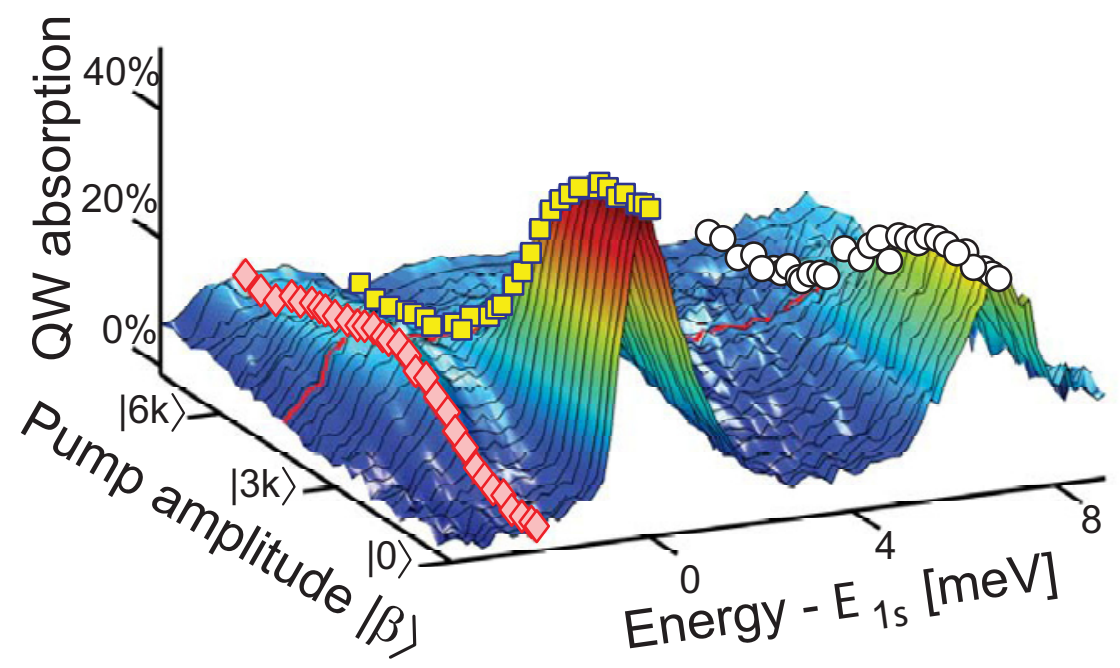

Fig. 1. Measured absorption spectra as a function of probe energy $E$ and pump amplitude $|\beta\rangle$. The $|\beta\rangle$ dependence of the absorption at the resonance energies of the biexciton $\left(2.25 \mathrm{meV}\right.$ below $E_{1 S}$, diamonds), the heavy-hole exciton $E_{1 S}=1.547 \mathrm{eV}$ (squares), and light-hole 1s-exciton energy (6.90 meV above $E_{1 S}$, circles).

the classical single-photon differential spectrum using the single-photon coherent state as a perturbation to a state with $12.5 \mathrm{M}$ photons, resulting in differential-absorption signals on the order of $10-8$, as seen in Fig. 2(a). This classical differential spectrum shows a negative dip around $E_{1 S}$, indicating that the 1S-absorption resonance broadens. This result is typical because the perturbation increases the electron-hole number and the excitation-induced scattering related to it.

Figure 2(b) presents the corresponding differential-absorption spectrum for where the perturbation is the replacement of one classical photon by a photon in a Schrödinger-cat state that has the appropriate phase relative to the coherent state so that the distribution function is squeezed in the radial direction. This perturbation enhances the nonlinear response at the biexciton, which occurs at -2.1 $\mathrm{meV}$ relative to the $1 \mathrm{~s}$ exciton, creating a narrower feature than is observed in the classical differential spectrum. As the number of classical photons is increased, the initial narrow biexciton resonance evolves into a broader resonance.

The observed features can be explained by the fact that the statistics of the light are mapped onto the electronic many-body state in the semiconductor by the absorption of the light. Thus quantum correlations in the light produce corresponding many-body correlations in the excited electron-hole pairs. Since a Schrödinger cat state represents two-photon correlations, the light state maps onto biexcitonic states, which are correlations between two electron-hole pairs. Higher-order correlations can also be
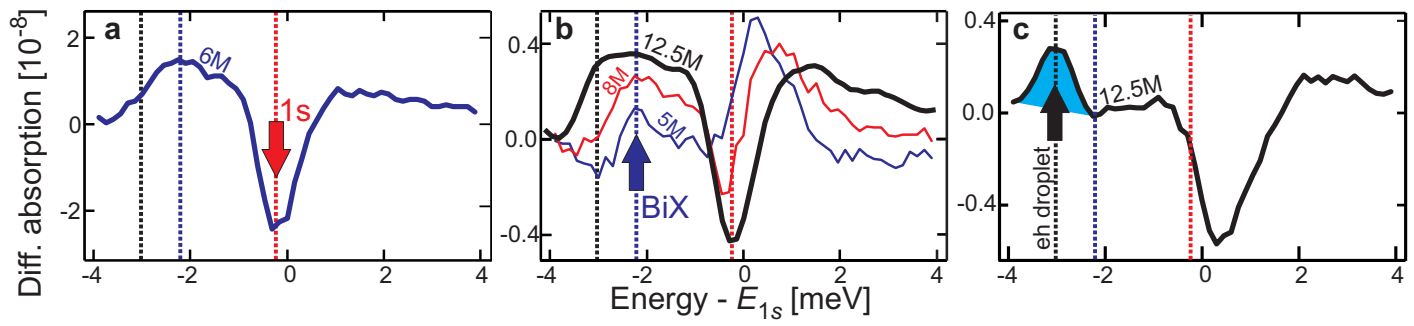

Fig. 2. Reconstructed differential absorption for a perturbation of (a) single classical photon, (b) single Schrödinger-cat photon, and (c) single asymmetric Schrödinger-cat photon. The dashed lines show the spectral positions of the biexciton and electron-hole droplet $(0.7 \mathrm{meV}$ below the biexciton resonance). 
observed. Figure 2(c) shows the differential spectrum when a photon corresponding to an asymmetric Schrödinger-cat state is used as the perturbation. This state consists of a coherent superposition of ordinary Schrödinger-cat states, and thus it has three-photon correlations. The resulting differential spectrum shows evidence for electron-hole droplets, which are more highly correlated than biexcitons.

These results demonstrate a new avenue to studying light-matter interactions, understanding how the quantum statistics of light map onto many-body excitations, and studying many-body dynamics. Future directions include using different quantum states of light, using $\mathrm{THz}$ fields and using more complex optical measurements, such as two-dimensional Fourier transform spectroscopy, to provide greater detail about the generated many-body state [5].

\section{References}

1. M. Kira and S.W. Koch, Phys. Rev. A 73, (2006) 013813

2. M. Kira, S.W. Koch, R.P. Smith, A.E. Hunter and S.T. Cundiff, Nature Physics. 7, (2011) 799

3. M. Kira and S.W. Koch, Phys Rev. A 78, (2008) 022102

4. R.P. Smith, J.K. Wahlstrand, A.C. Funk, R.P. Mirin, S.T. Cundiff, J.T. Steiner, M. Schafer, M.Kira and S.W. Koch, Phys. Rev. Lett. 104, (2010) 247401

5. S.T. Cundiff, J. Opt. Soc. Am. B 29, (2012) A69 